\title{
VARIANCE-REDUCED SIMULATION OF MULTISCALE TUMOR GROWTH MODELING*
}

\author{
ANNELIES LEJON ${ }^{\dagger}$, BERT MORTIER $^{\dagger}$, AND GIOVANNI SAMAEY ${ }^{\dagger}$
}

\begin{abstract}
We are interested in the mean-field evolution of a growing tumor as it emerges from a stochastic agent-based multiscale model. To this end, we introduce a hybrid PDE/Monte Carlo variance reduction technique. The variance reduction on the cell densities is achieved by combining a simulation of the stochastic agent-based model on the microscopic scale with a deterministic solution of a simplified (coarse) partial differential equation (PDE) on the macroscopic scale as a control variable. We show that this technique is able to significantly reduce the variance with only the (limited) additional computational cost associated with the deterministic solution of the coarse PDE. We illustrate the performance with numerical experiments in different biological scenarios.
\end{abstract}

Key words. Multiscale modeling, Tumor growth, Variance reduction

AMS subject classifications. 92-08, 62J10, 97M60

1. Introduction. Tumor growth is a complex biological phenomenon consisting of processes on different scales. On the cellular level - which will be referred to as the microscopic scale in this paper - one has to track the random motion of cells, as well as the cell division and cell death. The latter are governed by numerous intracellular processes. Furthermore, the cellular behavior is strongly coupled to the environment and vice versa. For example, local oxygen concentration and the local cell density are major contributors to the cell proliferation while hypoxia can trigger apoptosis. On the other hand, cells also consume oxygen [44, 26, 63]. This two-way feedback creates very specific dynamics characterizing the development of a tumor. A hypoxic zone develops in the middle of the tumor, which in turn triggers endothelial cells to vascularize the tumor. This process, also known as angiogenesis [11], ensures that the tumor's need for oxygen and other nutrients is satisfied, which facilitates further growth of the tumor.

Smaller avascular tumors can be easily simulated on the microscopic scale using agentbased models [37, 42, 4, 54, 61, 46]. We can distinguish two classes of models. On the one hand, cellular automata update grid cells based on a number of phenomenological rules, while on the other hand lattice-free models mainly consist of a set of ordinary differential equations (ODEs) attached to each cell.

On long time scales, we are mainly interested in the tumor as a whole, which we call the macroscopic scale. Agent-based models are typically not well suited to use on larger scales, since the individual based character implies a large computational cost for a large number of particles, corresponding to larger tumors. One may choose to model the system directly on this scale using continuum models, based on mass balance equations $[8,50,66,58,57,34]$. We refer to $[18,59,3]$ for a description of the use of such mean-field approaches to model large (biological) complex systems. While a mean-field approach results in significantly cheaper simulations than agent-based models, which are moreover easier to analyze, it cannot capture discrete features as branching of a vascular network or events regulated by intracellular concentrations.

\footnotetext{
* Submitted to the editors DATE.

Funding: The work of the corresponding author was funded by the IWT (Agency for Innovation by Science and Technology in Flanders) grant IWT-121616.

The second's author's work is funded by the Research Foundation - Flanders.

$\dagger$ Department of Computer Science, KU Leuven, Celestijnenlaan 200A, 3001 Leuven, Belgium (firstname.lastname@cs.kuleuven.be.
} 
Instead, the effects of such phenomena need to be modeled indirectly at the continuum scale, using approximate closure relations that describe the dynamics directly at the continuum level. For instance, a mean-field partial differential equation (PDE) that describes tumor density as a function of space and time needs to contain a reaction term that describes the increase in the number of tumor cells as a function of the tumor density, while tumor growth in reality arises due to complex intracellular dynamics that regulates the cell cycle.

The complementary requirements of a fast simulation method and sufficient modeling detail gave rise to multiscale models where agent-based models are typically used to model the cellular component, while the environment is mostly described by a set of reaction-diffusion partial differential equations, corresponding to the macroscopic scale. Examples can be found in [22, 44, 17, 30, 5, 43]. For a review about the current state of the art in multiscale modeling of tumor growth, we refer to [19, 48, 64, 13, 49]. A computational drawback of such agent-based models for the study of the mean-field behavior of tumor growth is their sensitivity to noise due to the random motion of the cells, which influences their interaction with the environment. When simulating with a standard Monte Carlo algorithm, the variance depends on the number of simulated cells. Various techniques for variance reduction such as antithetic variables, control variates and importance sampling are described in literature, see e.g. [9, 38] for an overview. Recently, several hybrid PDE/Monte Carlo algorithms have been proposed in the literature to achieve variance reduction by coupling a PDE-based discretization to a Monte Carlo simulation [20, 51, 47]. It is such a hybrid PDE/Monte Carlo method that we propose here for tumor growth.

In this paper, we aim at modeling mean-field behavior as accurately as possible for a reasonable computational cost, without explicitly deriving a closed form of the evolution on the macroscopic scale. Instead, we want to directly incorporate the influence of agent-based features on the mean-field level, which are not incorporated usually in mean field approximations. We start from a multiscale model that combines an agent-based description of the cells with a PDE-based description of the environment. However, we also introduce an approximate, mean-field PDE-based description of advection-diffusion type for the cellular component. This "coarse" approximation only accounts for the motion of the cells; no cell division of apoptosis is present at this level. However, the advection-diffusion behavior of the cells can be simulated deterministically with this model (thus without noise). This deterministic simulation is then augmented with the reaction term, which is computed directly from an agentbased simulation, without any closure approximation.

The contributions of this paper are two-fold:

- We develop a multiscale model where the random motion is modeled using stochastic differential equations (SDEs), the intracellular variables for the cell cycle and apoptosis are described by ODEs and the environment, consisting of diffusible components, is modeled by PDEs. The model is a modified version of the cellular automaton model of Owen et al. [44]. The main differences are that the new model is lattice-free and the fact that our model does not contain any explicit delay terms.

- We propose a novel technique to reduce the variance on the cellular densities obtained via an adapted multiscale agent-based model. Based on the ideas in $[51,20]$, we develop a hybrid PDE/Monte Carlo method using a coarse stochastic process (called the control process) and a corresponding PDE. In this specific case, the control process modeling the spatial behavior of the individual cells contains all details of the microscopic model except for cell 
births, cell deaths and VEGF (denoting Vascular Endothelial Growth Factor) secretion. The key point is to obtain this missing information with reduced variance by an appropriate coupling between the full microscopic agent-based model and the control process.

We first give a detailed overview of the different layers of the model in section 2 . Next, we describe the variance reduction algorithm in the section 3. We illustrate the technique numerically in section 4 . Finally, in section 5 we elaborate on a few possibilities for future research.

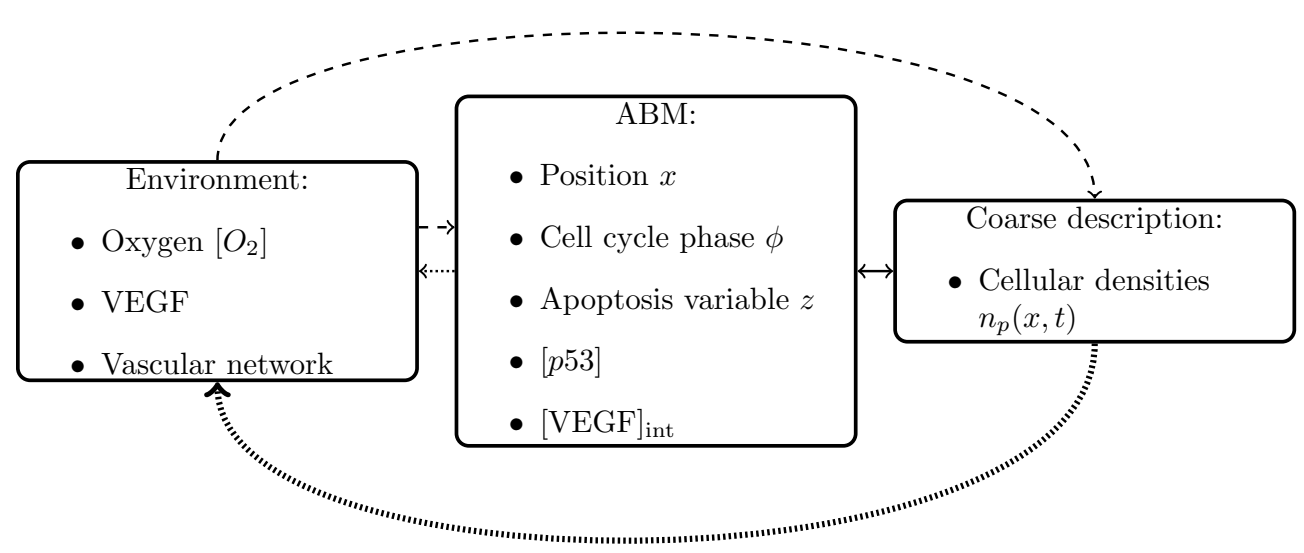

FIG. 1. Overview variables and interactions. Dashed lines indicates oxygen consumption; dotted lines denote VEGF production; the solid line denotes the fact that the density consists of cells.

2. Models. In this section, we describe a multiscale model for tumor growth. The microscopic model for tumor growth is based on the ideas used to describe bacterial chemotaxis [21,51], the multiscale cellular automaton model was developed by Owen and coworkers [44]. Our goal is to perform variance reduction in order to estimate the resulting population densities in a more accurate way. As in [51], the model proposed in this paper is time and space continuous. Apart from that the model is lattice-free, making the computational cost quasi independent of the size of the domain and hence, we can easily rescale the system to simulate larger tumors (compared to the examples given in [44]).

We distinguish two main components: the environment, modeled by a couple of reaction diffusion equations and the agent-based model describing the individual cellular motion and internal variables (e.g., cell cycle, apoptosis state and internal concentrations such as VEGF and the intracellular level of the tumor suppressor gene p53 [44]) attached to each cell.

We consider $P$ types of cells, indexed by $p=\{1,2, \ldots, P\}$. In this paper, we choose $P=3$, corresponding to normal cells $(p=1)$, cancer cells $(p=2)$, and endothelial cells (that build up blood vessels, $p=3$ ). For each of these cell types, we consider an ensemble of $I_{p}(t)$ cells, and consider three state variables: position $x \in \mathbb{R}^{2}$, cell cycle phase $\phi \in[0,1]$. The intracellular concentrations $[\mathrm{VEGF}]_{\mathrm{int}},[p 53]$ and apoptosis variable $z$ are scalars $\in \mathbb{R}$. These cells evolve according to evolution laws that depend on the concentration $\left[\mathrm{O}_{2}\right](\mathbf{x}, t)$ of oxygen and $[\mathrm{VEGF}](\mathbf{x}, t)$ of the Vascular Endothelial Growth Factor (which we call the environment).

Note that the reaction-diffusion partial differential equations (PDEs) describing the diffusible components of the environment still need to be solved on a grid, but this 
cost is marginal due to the sparsity of the involved linear systems, which ensures that the cost dependent on the domain size is limited.

We now give an overview of the notations that will be used throughout the paper, after which we describe the evolution laws for the environment, and detail the evolution laws for each of the cell types. The cell type dependency is mainly caused by cell type dependent coefficients, which will be discussed later on in the section describing the agent-based model in more detail.

Notation.

- Note that in general we use lower case letters for general variables and upper case letters to denote the actual value of a certain variable attached to a specific particle.

- The state variables attached to a single cell of type $p$ at time $t$ are position $\mathbf{X}_{p}(t)$, printed in bold to stress that we are dealing with a vector in this paper. The position variable $\mathbf{x}$ actually consists of two components $(x$ and $y)$. Further, cell cycle phase $\Phi_{p}(t)$, generation $\zeta_{p}(t)$, internal concentrations $[\mathrm{p} 53]_{p}(t),\left[\mathrm{VEGF}_{\mathrm{int}}\right]_{p}(t)$ and apoptosis variable $Z_{p}(t)$.

- Cell number densities are denoted by $n_{p}(\mathbf{x}, t)$ indexed by a suitable subscript to indicate the nature of the density. Further, $n_{v}(\mathbf{x}, t)$ is used to describe the vascular density.

- To keep a consistent notation throughout the paper, we introduce the following convention. If, at a moment $t=t^{\star}$, the cell with index $i^{\star}$ in population $p$ divides, we set

$$
I_{p}\left(t^{\star}\right)=I_{p}\left(t_{-}^{\star}\right)+1
$$

in which the symbol $t_{-}^{\star}$ is used to emphasize that the involved number of cells is meant to be taken just before the division. Simultaneously, we introduce a new cell. When a cell undergoes apoptosis, it is removed from the simulation. To avoid cumbersome renumbering of the cells in the text, we associate a weight $w_{i, p}(t)$ to each of the cells. If the cell is alive, the corresponding weight is one; upon apoptosis, it becomes zero. The active number of cells is therefore:

$$
\bar{I}_{p}(t)=\sum_{i=1}^{I_{p}(t)} w_{i, p}(t) .
$$

- The evolution of the state variables is influenced in various ways by the (local) environment. The latter will be modeled by means of diffusible components $[\mathrm{VEGF}](\mathbf{x}, t)$ describing the VEGF concentration, while $\left[O_{2}\right](\mathbf{x}, t)$ denotes the oxygen concentration.

- Throughout the text, we use multiple field quantities that depend on space and time, such as $n(\mathbf{x}, t)$ and $\left[O_{2}\right](\mathbf{x}, t)$. We will often need the values of these fields at particular particle position. Suppose that $f$ is a generic field quantity of this type, then $f\left(\mathbf{X}_{p}(t), t\right)$ represents the value of $f$ at the grid cell location corresponding to $\mathbf{X}_{p}(t)$ at time $t$.

2.1. Agent-based model. In this section, we give a detailed overview of the evolution of the different state variables attached to each cell of the different cellular populations. Note that there are multiple modeling strategies possible for each part of the agent-based model. For both cell division and apoptosis, we start from the intracellular model of Owen [44]. 
Position. The random motion of the position of the cells is described as a biased Brownian motion which is modeled via a stochastic differential equation (SDE) consisting of two parts: a random and a deterministic part. The infinitesimal change in position is denoted by $\mathrm{d} \mathbf{X}_{p}$. Cells of type $p$ move randomly with diffusion coefficient $D_{p}$, modeled via a standard Brownian motion with Brownian increments $\mathrm{d} \mathbf{W}_{t}$, and are chemotactically attracted towards high VEGF concentrations with sensitivity $\chi_{p}$. This sensitivity is only important for the endothelial cells, responsible for blood vessel growth,

$$
\mathrm{d} \mathbf{X}_{p}(t)=\underbrace{\left.\chi_{p} \nabla[\mathrm{VEGF}]\left(\mathbf{X}_{p}(t), t\right)\right)\left(1-\frac{n_{p}\left(\mathbf{X}_{p}(t), t\right)}{n_{\max , p}}\right) \mathrm{d} t}_{\text {Directed motion }}+\underbrace{\sqrt{2 D_{p}} \mathrm{~d} \mathbf{W}_{t}}_{\text {Random motion }} .
$$

in which the cell number density can be represented as:

$$
n_{p}(\mathbf{x}, t)=\sum_{i=1}^{I_{p}(t)} w_{i, p}(t) \delta_{\mathbf{X}_{i, p}(t)}
$$

where $I_{p}(t)$ denotes the total number of cells of type $p$ at time $t$ and $\delta_{\mathbf{X}_{i, p}(t)}$ is defined as follows:

$$
\delta_{\mathbf{X}_{i, p}(t)}= \begin{cases}1 & \mathbf{X}_{i, p}(t) \in \text { grid cell } \mathbf{x} \\ 0 & \text { otherwise }\end{cases}
$$

Note that, in contrast to the cellular automaton model with discrete space jumps described in [44], the resulting equation for the position is a stochastic differential equation (SDE). Finally, we stress that the above equation is general for all the cell types and differences between cell types are induced by specific choices of parameters. For instance, normal cells don't move at all, while cancer cells are characterized by pure diffusive motion and endothelial cells demonstrate diffusive behavior while also responding to chemotactic cues.

Cell division. This process is modeled by means of the following ODE:

$$
\frac{\mathrm{d} \Phi_{p}(t)}{\mathrm{d} t}=\underbrace{\frac{\left[O_{2}\right]\left(\mathbf{X}_{\mathbf{p}}(t), t\right)}{\tau_{\min , p}\left(C_{\phi, p}+\left[O_{2}\right]\left(\mathbf{X}_{\mathbf{p}}(t), t\right)\right)}}_{\text {Cell cycle speed on local oxygen concentration }} \times \underbrace{\mathrm{H}\left(\zeta_{p, \max }-\zeta_{p}(t)\right)}_{\text {Restriction depending on generation }},
$$

where $\tau_{\min , p}$ denotes the minimal time needed for a cell to complete one cell cycle, $\mathrm{H}$ defines the classical Heaviside function and hence we have the following equation:

$$
\mathrm{H}\left(\zeta_{p, \max }-\zeta_{p}(t)\right)= \begin{cases}1 & \text { if } \zeta_{p}(t) \leq \zeta_{p, \max } \\ 0 & \text { otherwise }\end{cases}
$$

and $\zeta$ indicates the generation of a cell. Note that $\tau_{\min , p}$ depends on the cell type. To be more specific, cancer cells are able to proceed twice as fast as normal cells during the cell cycle in a given environment (see table 1). Naturally, the cell cycle speed depends on the local oxygen concentration $\left[O_{2}\right]\left(\mathbf{X}_{p}(t), t\right)$ as observed by the cell while evolving through the cycle. The higher the oxygen concentration, the faster the cycle is completed, while the cell cycle is put on hold when the cell suffers from hypoxia. A more detailed biological motivation for this model can be found in [44, 62] and its 
supplementary material. Note that in the cellular automaton model by Owen et al. (see [44]), all cells can divide an unlimited number of times, which is obviously not a realistic assumption. Thus, we have extended the model to account for the fact that cells are only able to divide a finite number of times (i.e. $\zeta_{p, \max }$ ). To be more specific we added a factor $\mathrm{H}\left(\zeta_{p, \max }-\zeta_{p}(t)\right)$ to check for the generation of the corresponding cells. Here, we assume that normal cells can divide only 4 times, which is consistent with [23]. On the other hand we assume that all the cancer cells are cancer stem cells, which is still a simplification.

If, for the cell with index $i^{\star}$ in population $p$ at time $t=t^{\star}$, we obtain $\Phi_{i^{\star}, p}\left(t^{\star}\right) \geq 1$ and $\zeta_{i^{\star}, p}\left(t^{\star}\right) \leq \zeta_{\max }$, we introduce a new cell in the simulation. We adjust $I_{p}(t)$ according to equation (1) and set $\Phi_{i^{\star}, p}(t)=0$. and the generation of the parent cell increases by one. The new cell inherits the state from the cell that divides except for the generation $\zeta$ :

$$
\begin{aligned}
\mathbf{X}_{I_{p}(t), p}\left(t^{\star}\right) & =\mathbf{X}_{i^{\star}, p}\left(t^{\star}\right), & Z_{I_{p}(t), p}\left(t^{\star}\right) & =Z_{i^{\star}, p}\left(t^{\star}\right), \\
{[\mathrm{p} 53]_{I_{p}(t), p}\left(t^{\star}\right) } & =[\mathrm{p} 53]_{i^{\star}, p}\left(t^{\star}\right), & {\left[\mathrm{VEGF}_{\mathrm{int}}\right]_{I_{p}(t), p}\left(t^{\star}\right) } & =\left[\mathrm{VEGF}_{\mathrm{int}}\right]_{i^{\star}, p}\left(t^{\star}\right), \\
\Phi_{I_{p}(t), p}\left(t^{\star}\right) & =\Phi_{i^{\star}, p}\left(t^{\star}\right), & \zeta_{I_{p}(t), p}\left(t^{\star}\right) & =0 .
\end{aligned}
$$

Intracellular model. We introduce a intracellular module consistent with [44] in order to describe some important intracellular concentrations, namely the p53 concentration [ $p 53]$ and the intracellular VEGF concentration [VEGF $i n t]$. We have:

$$
\begin{aligned}
\frac{\mathrm{d}[\mathrm{p} 53]_{p}(t)}{\mathrm{d} t} & =c_{1}-c_{2} \frac{\left[O_{2}\right]\left(\mathbf{X}_{p}(t), t\right)}{C_{p 53}+\left[O_{2}\right]\left(\mathbf{X}_{p}(t), t\right)}[\mathrm{p} 53]_{p}(t), \\
\frac{\mathrm{d}\left[\mathrm{VEGF}_{\mathrm{int}}\right]_{p}(t)}{\mathrm{d} t} & =c_{3}-c_{4} \frac{[\mathrm{p} 53]_{p}(t)\left[\mathrm{VEGF}_{\mathrm{int}}\right]_{p}(t)}{J_{5}+\left[\mathrm{VEGF}_{\mathrm{int}}\right]_{p}(t)} \\
& -c_{5} \frac{\left[O_{2}\right]\left(\mathbf{X}_{p}(t), t\right)}{C_{\mathrm{VEGF}}+\left[O_{2}\right]\left(\mathbf{X}_{p}(t), t\right)}\left[\mathrm{VEGF}_{\mathrm{int}}\right]_{p}(t)
\end{aligned}
$$

Cells are storing VEGF intracellular (i.e. [VEGF $\left.{ }_{\text {int }}\right]$ ) during hypoxic conditions and release it once this intracellular concentration has reached a certain threshold level $\left[\mathrm{VEGF}_{\text {int }}\right]_{\mathrm{thr}}$. Further, $c_{1}, \ldots c_{5}$ and $C_{\mathrm{p} 53}, C_{\mathrm{VEGF}}$ are constants that can be found in table 1. Next we describe the model for apoptosis, depending on the cell type. Therefore we formally define $\gamma_{\text {apt }, p}\left(z, n_{p}\right)=F_{p}\left(z, n_{p}\right)$ as the apoptosis rate, which is further specified in the following paragraphs.

Apoptosis for normal cells. Cell death can be modeled in many ways $[52,24,6,56]$. Our approach is based on the work of Owen et al. [44]. For normal cells, cell death is completely determined by the intracellular p53-concentration. So, we set the apoptosis variable $z:=[p 53]$. The apoptosis threshold $\gamma_{\text {apt }, 1}\left(z, n_{1}\right)$ can then be written as:

$$
\gamma_{\text {apt }, 1}\left(z, n_{1}\right)=\mathrm{H}(z-\underbrace{z_{\text {low }} \mathrm{H}\left(n_{\mathrm{thr}}-n_{1}\right)}_{\text {Harsh environment }}-\underbrace{z_{\mathrm{high}} \mathrm{H}\left(n_{1}-n_{\mathrm{thr}}\right)}_{\text {Normal environment }}) \text {, }
$$

where $\mathrm{H}$ indicates the Heaviside function. This definition of $\gamma_{\mathrm{apt}, 1}$ implies that normal cells undergo apoptosis if $\gamma_{\mathrm{apt}, 1}\left(z, n_{1}\right)=1$ corresponding to the situation that $z$ has reached a certain threshold value depending on the harshness of the environment. The threshold value is lower in case of a harsh environment, defined as $n_{1}<n_{\mathrm{thr}}$, where $n_{\text {thr }}$ denotes a threshold value for the normal cells. 
Apoptosis for cancer cells. In contrast to normal cells, the apoptosis mechanism for tumor cells is independent of the p53-concentration since this mechanism to regulate the normal cell cycle, is assumed to be malfunctioning as is the case in many tumors $[2,30,65]$. The cancer cells in our model are able to go into a quiescent state when expressed to hypoxic circumstances, meaning that they don't consume any nutrients anymore for a while. However the duration of this quiescent state is limited, which implies that cancer cells will also undergo apoptosis when the hypoxia holds too long. On the other hand, cancer cells have the ability to recover quickly once there is again more oxygen available. This mechanism can be modeled by the following equation:

$$
\frac{\mathrm{d} Z(t)}{\mathrm{d} t}=\underbrace{A \mathrm{H}\left(\left[\mathrm{O}_{2}\right]_{\mathrm{thr}}-\left[\mathrm{O}_{2}\right]\left(\mathbf{X}_{p}(t), t\right)\right)}_{\text {Linear increase during hypoxia }}-\underbrace{B Z(t) \mathrm{H}\left(\left[\mathrm{O}_{2}\right]\left(\mathbf{X}_{p}(t), t\right)-\left[O_{2}\right]_{\mathrm{thr}}\right)}_{\text {Exponential decay if }\left[O_{2}\right]\left(\mathbf{X}_{p}(t), t\right)>\left[O_{2}\right]_{\mathrm{thr}}},
$$

where $A, B$ are constants. The first term models the hypoxic state, i.e. the local oxygen concentration $\left[O_{2}\right]\left(\mathbf{X}_{p}(t), t\right)$ drops below the threshold level $\left[O_{2}\right]_{\text {thr }}$. During this hypoxic period, the internal variable $z$ increases steadily. On the other hand, the second term describes the recovery of the cancer cells if the environment is not hypoxic anymore, which is captured by the exponential decay term of $Z(t)$. Cancer cells die if $Z(t) \geq 1$, corresponding to $\gamma_{\text {apt }, 2}(z)=\mathrm{H}(z-1)$.

Endothelial cells. Note that the model equations concerning cell division and cell death will not be used for endothelial cells. Consistent with existing literature, the so-called snail-trail approach is used to model sprouting angiogenesis [7, 32].

Full agent-based model. This results in the following set of equations for the full agent-based model:

(11)

$$
\left\{\begin{array}{l}
\left.d X_{p}(t)=\chi_{p} \nabla[\operatorname{VEGF}]\left(\mathbf{X}_{p}(t), t\right)\right)\left(1-\frac{n_{p}\left(\mathbf{X}_{p}(t), t\right)}{n_{\max , p}}\right) \mathrm{d} t+\sqrt{2 D_{p}} \mathrm{~d} \mathbf{W}_{t}, \\
\frac{\mathrm{d} \Phi_{p}(t)}{\mathrm{d} t}=\frac{\left[O_{2}\right]\left(\mathbf{X}_{p}(t), t\right)}{\tau_{\min , p}\left(C_{\phi, p}+\left[O_{2}\right]\left(\mathbf{X}_{p}(t), t\right)\right)} \mathrm{H}\left(\zeta_{p}(t)-\zeta_{p, \max }\right), \\
\frac{\mathrm{d}[\mathrm{p} 53]_{p}(t)}{\mathrm{d} t}=c_{1}-c_{2} \frac{\left[O_{2}\right]\left(\mathbf{X}_{p}(t), t\right)}{C_{p 53}+\left[O_{2}\right]\left(\mathbf{X}_{p}(t), t\right)}[\mathrm{p} 53]_{p}(t), \\
\frac{\mathrm{d}\left[\mathrm{VEGF}{ }_{\text {int }}\right]_{p}(t)}{\mathrm{d} t}=c_{3}-c_{4} \frac{[\mathrm{p} 53]_{p}(t)\left[\mathrm{VEGF}_{\mathrm{int}}\right]_{p}(t)}{J_{5}+\left[\mathrm{VEGF}_{\mathrm{int}}\right]_{p}(t)}+c_{5} \frac{\left[O_{2}\right]\left(\mathbf{X}_{p}(t), t\right)}{C_{\mathrm{VEGF}}+\left[O_{2}\right]\left(\mathbf{X}_{p}(t), t\right)}\left[\mathrm{VEGF}_{\mathrm{int}}\right]_{p}(t), \\
\frac{\mathrm{d} Z_{1}}{\mathrm{~d} t}=\frac{\mathrm{d}[p 53]}{\mathrm{d} t} \\
\frac{\mathrm{d} Z_{2}}{\mathrm{~d} t}=A \mathrm{H}\left(\left[O_{2}\right]_{\mathrm{thr}}-\left[O_{2}\right]\left(\mathbf{X}_{p}(t), t\right)\right)-B Z(t) \mathrm{H}\left(\left[O_{2}\right]\left(\mathbf{X}_{p}(t), t\right)-\left[O_{2}\right]_{\mathrm{thr}}\right) \\
\gamma_{\mathrm{apt}, p}\left(z, n_{p}, t\right)=F_{p}\left(z, n_{p}, t\right) .
\end{array}\right.
$$

Based on the supplementary material [44], we choose the parameter values as in table 1.

2.2. Mean-field description. An alternative approach to model tumor growth is to describe the evolution of the cell densities using partial differential equations 


\begin{tabular}{|c|c|c|c|c|}
\hline Parameter & $n_{1}$ & $n_{2}$ & $n_{3}$ & units \\
\hline$\chi_{p}$ & 0.0 & 0.0 & $2 \times 10^{-4}$ & $\mathrm{~cm}^{2} / \mathrm{min} / \mathrm{nM}$ \\
\hline$\zeta_{p, \max }$ & 4 & $\infty$ & 4 & times \\
\hline$C_{\phi, p}$ & 3 & 1.4 & & $\mathrm{mmHg}$ \\
\hline$C_{\mathrm{VEGF}}$ & 0.01 & 0.01 & 0.01 & $\mathrm{mmHg}$ \\
\hline$C_{\mathrm{p} 53}$ & 0.01 & 0.01 & 0.01 & $\mathrm{mmHg}$ \\
\hline$\tau_{p, \min }$ & 3000 & 1600 & & $\min$ \\
\hline$z_{\text {high }}$ & 0.8 & & & dimensionless \\
\hline$z_{\text {low }}$ & 0.08 & & & dimensionless \\
\hline$n_{\mathrm{thr}}$ & 0.75 & & & dimensionless \\
\hline$\left[\mathrm{O}_{2}\right]_{\mathrm{thr}}$ & & 8.9 & & $\mathrm{mmHg}$ \\
\hline$\left[\mathrm{VEGF}_{\text {int }}\right]_{\mathrm{thr}}$ & 0.27 & 0.27 & & $\mathrm{nM}$ \\
\hline$c_{1}$ & $2 \times 10^{-3}$ & $2 \times 10^{-3}$ & & $\min ^{-1}$ \\
\hline$c_{2}$ & $1 \times 10^{-2}$ & $1 \times 10^{-2}$ & & $\min ^{-1}$ \\
\hline$c_{3}$ & $2 \times 10^{-3}$ & $2 \times 10^{-3}$ & & $\min ^{-1}$ \\
\hline$c_{4}$ & $2 \times 10^{-3}$ & $2 \times 10^{-3}$ & & $\min ^{-1}$ \\
\hline$c_{5}$ & $1 \times 10^{-2}$ & $1 \times 10^{-2}$ & & $\min ^{-1}$ \\
\hline$J_{5}$ & 0.04 & 0.04 & & $\mathrm{nM}$ \\
\hline$A$ & & 1 & & $\min ^{-1}$ \\
\hline$B$ & & $2.5 \times 10^{-3}$ & & $\min ^{-1}$ \\
\hline
\end{tabular}

Parameter values related to the populations, chosen in agreement with [44].

(PDEs). In general, this approach yields a reaction-diffusion PDE of the following form:

$(12)$

$\partial_{t} n_{p}(\mathbf{x}, t)=D_{p} \nabla^{2} n_{p}(\mathbf{x}, t)-\chi_{p} \nabla \cdot\left[n_{p}(\mathbf{x}, t)\left(1-\frac{n_{p}(\mathbf{x}, t)}{n_{p, \max }}\right) \nabla[\operatorname{VEGF}](\mathbf{x}, t)\right]+R(\mathbf{x} . t)$,

where the reaction term $R(\mathbf{x}, t)$ accounts for the (dis)appearance of cells (i.e., cell division and apoptosis). A closed (mean-field) model would require one to write $R(\mathbf{x}, t)$ in terms of the densities $n_{p}(\mathbf{x}, t)$. However, in this case, obtaining such a closed formulation is not possible, since cell division and apoptosis depends on intracellular processes. Nevertheless, formally, a simulation of a large number of cells, using the agent-based model outlined in section 2.1, can be seen as a particle-based discretization of the above mean-field PDE, even if it is not available in closed form.

When one ignores births and deaths, obtaining a closed model at the level of cell densities is possible, see [33]. The resulting macroscopic equation for the evolution of the populations is of advection-diffusion type and reads:

$$
\partial_{t} n_{p}(\mathbf{x}, t)=D_{p} \nabla^{2} n_{p}(\mathbf{x}, t)-\chi_{p} \nabla \cdot\left[n_{p}(\mathbf{x}, t)\left(1-\frac{n_{p}(\mathbf{x}, t)}{n_{p, \max }}\right) \nabla[\operatorname{VEGF}](\mathbf{x}, t)\right],
$$

and is obtained by taking the limit of the number of cells going to infinity.

From now on, we will consider the agent-based model of section 2.1 as a stochastic particle discretization of the mean-field model (12) that is not available in closed form. We will therefore interchangeably use the terms "particle" and "cells" in the remainder of the manuscript when we denote instances of the stochastic process outlined in section 2.1 . 
Finally, we also introduce the following discretization of the advection-diffusion PDE (13):

$$
\begin{aligned}
n_{p}\left(\mathbf{x}, t^{k+1}\right)= & n_{p}\left(\mathbf{x}, t^{k}\right)+\delta t D_{p} \nabla^{2} n_{p}\left(\mathbf{x}, t^{k}\right) \\
& -\delta t\left(\chi_{p} \nabla \cdot\left[n_{p}\left(\mathbf{x}, t^{k}\right)\left(1-\frac{\left.n_{p}\left(\mathbf{x}, t^{k}\right)\right)}{n_{p, \max }}\right) \nabla[\operatorname{VEGF}]\left(\mathbf{x}, t^{k}\right)\right]\right),
\end{aligned}
$$

in which $\delta t$ denotes the time-step and we have used a first order Euler discretization to discretize the time derivative and a second order central finite volume scheme to discretize the spatial derivatives. Further details can be found in section 4 .

2.3. Angiogenesis. The growth of new blood vessels, also known as angiogenesis, is essential for the further development of a tumor. Hanahan and co-authors identified it as one of the hallmarks of cancer (see [30, 29]). For a general review about angiogenesis, we refer to the work of Carmeliet et al. [12]; specifically for tumor angiogenesis, we mention $[40,14]$. In the early stages of cancer, the existing vasculature is able to provide a sufficient amount of oxygen and other nutrients. However, as soon as the size of the tumor has reached a certain threshold, a hypoxic zone develops in the middle of the tumor $[39,55]$. To cope with this phenomenon, the tumor secretes VEGF, a growth factor, which triggers endothelial cells to move chemotactically towards this hypoxic zone and grow new blood vessels. In this paper we will use an existing model for angiogenesis, described in [44]. We distinguish two phenotypes: endothelial cells can either be motile leader cells (also called tip cells) or static stalk cells. We model proliferation of endothelial cells by means of the so-called snail-trail approach, where each tip cell produces a new (static) endothelial cell at its previous position, creating a trail of static stalk cells behind him. Apart from this feature, new tip cells -known as sprouts - can emerge from active vessels with sprouting probability $P_{\text {sprout }}$ along the active vessels. (see [44]):

$$
P_{\text {sprout }}=\delta t \frac{P_{\max }[\mathrm{VEGF}](\mathbf{x}, t)}{V_{\text {sprout }}+[\mathrm{VEGF}](\mathbf{x}, t)}
$$

where $P_{\max }=3 \times 10^{-4} \min ^{-1}$ indicates the maximal endothelial sprouting rate ( see [44]) and $V_{\text {sprout }}=0.5 \mathrm{nM}$ denotes the VEGF concentration at which the sprouting probability is half maximal. Note that the probability of the emergence of two sprouts close to each other within the same time-step is zero. A biological explanation for this fact can be found in the supplementary material provided with [44] and $[27,7,35]$, where the authors pointed out that delta-notch signaling inhibits the formation of new sprouts in neighboring endothelial cells. Additionally, the vessel radii are also adapted dynamically based on the work of [44] where pruning of the vessels was also incorporated in the model: if the pressure in a branch is too low, the corresponding branch will collapse.

2.4. Environment. We model the cellular environment by means of two diffusible components regulating the behavior of the cells in various ways. Oxygen is evidently important for the cells to proceed through the cell cycle [36, 44, 48, 1, 45]. 
The local oxygen concentration is determined from the following equation:

$$
\begin{aligned}
\partial_{t}\left[O_{2}\right](\mathbf{x}, t)= & \underbrace{D_{\left[O_{2}\right]} \nabla^{2}\left[O_{2}\right](\mathbf{x}, t)}_{\text {diffusion }}+\underbrace{\psi_{\left[O_{2}\right]} n_{v}(\mathbf{x}, t)\left(\left[O_{2}\right]_{\text {blood }}-\left[O_{2}\right](\mathbf{x}, t)\right)}_{\text {exchange with blood }} \\
& +\underbrace{\left[O_{2}\right](\mathbf{x}, t) k_{\left[O_{2}\right]} \sum_{p=1}^{P} n_{p}(\mathbf{x}, t)}_{\text {Consumption }},
\end{aligned}
$$

where $D_{\left[\mathrm{O}_{2}\right]}$ is the diffusion coefficient of oxygen, $\psi_{\left[\mathrm{O}_{2}\right]}$ denotes the permeability of the oxygen through the vessels. $n_{v}(\mathbf{x}, t)$ describes the surface area occupied by the vessel at position x. $\left[\mathrm{O}_{2}\right]_{\text {blood }}(\mathbf{x}, t)=\left[\mathrm{O}_{2}\right]_{\mathrm{ref}} H(\mathbf{x}, t) / H_{\text {in }}$ defines the oxygen concentration in a blood vessel located at position $\mathbf{x}$. $\left[\mathrm{O}_{2}\right]_{\text {ref }}$ is a reference oxygen concentration, $H(\mathbf{x}, t)$ is the haematocrit at location $\mathbf{x}$ and time $t$ and $H_{\text {in }}$ is the haematocrit at an inflow node. In agreement with [44], we set this value by default to $H_{\text {in }}=0.45$. The last term in (16) reflects the fact that all cells consume oxygen at a rate $k_{\left[\mathrm{O}_{2}\right]}$.

A similar approach is used to describe the local concentration of growth factors (e.g., Vascular Endothelial Growth Factors) denoted by [VEGF]. VEGF plays a crucial role in vascular tumor growth, since it triggers angiogenesis. Hence, it is usually taken into account in recent tumor growth modeling [10,53]. The corresponding reaction-diffusion equation for VEGF reads:

(17)

$$
\begin{aligned}
\partial_{t}[\mathrm{VEGF}](\mathbf{x}, t)= & \underbrace{D_{\mathrm{VEGF}} \nabla^{2}[\mathrm{VEGF}](\mathbf{x}, t)}_{\text {diffusion }}-\underbrace{\psi_{\mathrm{VEGF}} n_{v}(\mathbf{x}, t)[\mathrm{VEGF}](\mathbf{x}, t)}_{\text {decay }}+\underbrace{S_{\mathrm{VEGF}}(\mathbf{x}, t)}_{\text {production }}, \\
& -\underbrace{\delta_{\mathrm{VEGF} \operatorname{VEGF}(\mathbf{x}, t)}}_{\text {vith blood }} \\
S_{[\mathrm{VEGF}]}(\mathbf{x}, t)= & k_{[\mathrm{VEGF}]} \sum_{p=1}^{P} \sum_{i=1}^{I_{p}(t)} \delta_{\mathbf{x}_{i, p}(t)} \mathrm{H}\left(\left[\mathrm{VEGF}_{\mathrm{int}}\right]_{i, p}(t)-\left[\mathrm{VEGF}_{\mathrm{int}}\right]_{\mathrm{thr}}\right) .
\end{aligned}
$$

\begin{tabular}{|c|c|c|c|}
\hline Parameter & Oxygen & VEGF & units \\
\hline$D$ & 0.0014 & $6 \times 10^{-4}$ & $\mathrm{~cm}^{2} / \mathrm{min}$ \\
$\psi$ & 6 & $6 \times 10^{-4}$ & $\mathrm{~cm}^{-m i n}$ \\
$\delta$ & 0 & 0.6 & $\mathrm{~min}^{-1}$ \\
$k_{\left[\mathrm{O}_{2}\right]}$ & -13 & & $\mathrm{~min}^{-1}$ \\
$\left.k_{[\mathrm{VEGF}}\right]$ & & 0.6 & $\mathrm{~min}^{-1}$ \\
{$\left[\mathrm{O}_{2}\right]_{\text {ref }}$} & 20 & & $\mathrm{mmHg}$ \\
\hline
\end{tabular}

Parameter values of the reaction diffusion equations.

3. Variance reduction. In this section, we propose an algorithm to reduce the variance on the cell densities $n_{p}(\mathbf{x}, t)$ obtained from the agent-based simulation. The algorithm relies on the combination of three simulations: (i) a stochastic simulation with the full microscopic model; (ii) a coupled stochastic simulation with an approximate, coarse agent-based model; and (iii) a deterministic grid-based simulation of the same coarse model. The full microscopic model uses an ensemble of $I_{p}(t)$ particles with state variables:

$$
\left\{\mathbf{X}_{i, p}(t), Z_{i, p}(t), \Phi_{i, p}(t), \zeta_{i, p}(t),[\mathrm{p} 53]_{i, p}(t),\left[\mathrm{VEGF}_{\mathrm{int}}\right]_{i, p}(t)\right\}_{i=1}^{I_{p}(t)} .
$$


As the coarse agent-based model, we conceptually consider an agent-based model in which the internal state has been suppressed and only the position remains:

$$
\left\{\mathbf{X}_{i, p}^{c}(t)\right\}_{i=1}^{I_{p}(t)} .
$$

So, no internal dynamics is present in the coarse model: cells cannot divide, die or secrete VEGF. (In practice, we will not perform a separate simulation for the coarse model, but instead use the results obtained from the complete agent-based model, in which we neglect the effects of apoptosis and cell division). The only dynamics remaining then is motion, which can be modeled with a PDE for the population density (see equation (13)). We call this coarse approximation the control process. We also introduce the formal semi-group notation:

$$
e^{t L_{p}^{c}\left(n_{p}^{c}\right)} \quad \text { with } L_{p}^{c}\left(n_{p}^{c}\right)=-D_{p} \nabla^{2}-\chi_{p} \nabla \cdot\left[n_{p}(\mathbf{x}, t)\left(1-\frac{n_{p}(\mathbf{x}, t)}{n_{p, \max }} \nabla[\operatorname{VEGF}](\mathbf{x}, t)\right)\right],
$$

that represents the exact solution of the macroscopic partial differential equation (13). In practice, the solution will be approximated by a deterministic solution on a grid. It should be clear that the advection-diffusion behavior in both agent-based models is identical. Thus, the only difference between the two models occurs when cells divide or die. Assuming no reactions take place, the three processes thus have the same expectation. This observation leads to the following variance reduction algorithm. As an initial condition, we start from $I_{p}(0)$ particles representing the number density $n_{p}(x, 0)$. For each particle, we choose a given internal state, for instance $\Phi_{i, p}(0)=$ $Z_{i, p}(0)=0, \zeta_{i, p}(0)=0,[p 53]_{i, p}(0)=0,\left[\mathrm{VEGF}_{\mathrm{int}}\right]_{i, p}(0)=0,1 \leq i \leq I_{p}(0), 1 \leq$ $p \leq P$. (These internal states could also be sampled from an appropriate probability distribution.) Additionally, we introduce the variance reduced measure $\bar{n}_{p}(\mathbf{x}, t)$, which we initialize as $\bar{n}_{p}(\mathbf{x}, 0)=n_{p}(\mathbf{x}, 0)$. We denote the time step $\delta t$ and the discrete time instances $t^{\ell}=\ell \delta t, \ell \geq 0$.

AlGORITHM 1 (Variance reduction for tumor growth). We advance the variance reduced number density $\bar{n}(\mathbf{x}, t)$ from time $t^{\ell}$ to $t^{\ell+1}$ as follows:

- Evolve the particle states (18) from $t^{\ell}$ to $t^{\ell+1}$ using the agent-based model (11).

- Compute the number density for the stochastic microscopic model using (4), as well as the number density for the coarse process as

$$
n_{p}^{c}\left(\mathbf{x}, t^{\ell+1}\right)=\sum_{i=1}^{I_{p}\left(t^{\ell}\right)} w_{i, p}\left(t^{\ell}\right) \delta_{\mathbf{X}_{i, p}\left(t^{\ell+1}\right)},
$$

i.e., we compute the number density for the control process based on particle positions and velocities at time $t^{\ell+1}$, taking into account only the particles that were present in the simulation at time $t^{\ell}$.

- Evolve the control number density $n_{p}^{c}(\mathbf{x}, t)$ using a grid-based method based on (13) and add the reactions (the difference in number density due to cell division and apoptosis)

$$
\bar{n}_{p}\left(\mathbf{x}, t^{\ell+1}\right):=\bar{n}_{p}\left(\mathbf{x}, t^{\ell}\right) e^{\delta t L^{c}}+n_{p}\left(\mathbf{x}, t^{\ell+1}\right)-n_{p}^{c}\left(\mathbf{x}, t^{\ell+1}\right) .
$$

This procedure will be repeated after reinitializing the control density $\tilde{n}_{p}(\mathbf{x}, t)=$ $\bar{n}_{p}(\mathbf{x}, t)$. Reinitialization guarantees that these differences between the full and the 
coarse model do not accumulate over time. The importance of reinitialization can be illustrated by looking into the following hypothetical situation. Suppose the $i$ th cell of type $p$ divides at time $t=t^{\star}$, and hence cell $I_{p}+1$ is born. At time $t>t^{\star}$, this newborn cell has moved randomly through the domain. Apart from this random motion, it also has influenced the environment along its track.

The algorithm can alternatively be written in terms of the reaction field:

DEFINITION 2 (Reaction field). The control process differs from the full microscopic model in the way that there are no births, deaths or VEGF-secretion events. The direct influence on the population density can be summarized by the reaction field defined as:

$$
\begin{aligned}
R_{p}\left(\mathbf{x}, t^{l+1}\right) & =n_{p}\left(\mathbf{x}, t^{l+1}\right)-n_{p}^{c}\left(\mathbf{x}, t^{l+1}\right) \\
& =\sum_{i=1}^{I_{p}\left(t^{l+1}\right)} w_{i, p}\left(t^{l+1}\right) \delta_{\mathbf{X}_{i, p}\left(t^{l+1}\right)}-\sum_{i=1}^{I_{p}\left(t^{l}\right)} w_{i, p}\left(t^{l}\right) \delta_{\mathbf{X}_{i, p}\left(t^{l+1}\right)} .
\end{aligned}
$$

We also introduce a shorthand notation:

Definition 3 (Deterministic control density). Denote the control density calculated with the macroscopic evolution equation as:

$$
\tilde{n}_{p}\left(\mathbf{x}, t^{l+1}\right):=\bar{n}_{p}(\mathbf{x}, t) e^{\delta t L^{c}}
$$

To see that the proposed estimator for the population densities $n_{p}$ is unbiased and that the algorithm indeed reduces the variance on the cell densities, we have the following theorem:

THEOREM 4 (Unbiased estimator). Assume that discretization errors are absent. Then, the algorithm described above yields an unbiased estimator for the population density $n_{p}$.

Proof. Assume that discretization errors are absent. Then, we can calculate the expectation value of $\bar{n}_{p}$ based on equation (22) as follows:

$$
\mathbb{E}\left[\bar{n}_{p}\left(\mathbf{x}, t^{l+1}\right)\right]=\mathbb{E}\left[\tilde{n}_{p}\left(\mathbf{x}, t^{l+1}\right)\right]+\mathbb{E}\left[n_{p}\left(\mathbf{x}, t^{l+1}\right)\right]-\mathbb{E}\left[n_{p}^{c}\left(\mathbf{x}, t^{l+1}\right)\right],
$$

where $\mathbb{E}\left(n_{p}(\mathbf{x}, t)\right)$ denotes the expectation value of the quantity $n_{p}(\mathbf{x}, t)$. By using sequentially the definition of $\tilde{n}_{p}^{c}$ (see equation (24)) and the linearity of $\mathbb{E}$, we can conclude that $\mathbb{E}\left[\bar{n}_{p}\right]=\mathbb{E}\left[\tilde{n}_{p}\right]$ and hence $\bar{n}_{p}$ is indeed an unbiased estimator.

Note that the algorithm described above is similar to the technique used in [51] to simulate bacterial chemotaxis. The main differences are due to the fact that (i) the model is not conservative; and (ii) the internal dynamics only relates to cell division, apoptosis and VEGF secretion and not to advection-diffusive behavior.

4. Results. In this section, we will illustrate the performance of the variance algorithm described above with various numerical experiments. The cells are living on a $50 \times 50$ square grid. By default 2000 normal cells are uniformly distributed over the whole domain. A small tumor consisting of 200 cancer cells are initially normally distributed with mean $0.25 \Delta x$ and standard deviation $0.05 \Delta x$ closely to the left vessel. We simulate the system over 1920 time-steps (or 40 days). The whole set of default parameters is summarized in table 3 . To initialize the agent-based simulation, we sampled $I_{p}(0)$ particles from the corresponding distribution. As pointed out before, 
we are mainly interested in the evolution of a probability distribution. Hence, the equation to calculate the cell number density can be rewritten as:

$$
n_{p}(\mathbf{x}, t)=\sum_{i=1}^{I_{p}(t)} q_{i, p} w_{i, p}(t) \delta_{\mathbf{X}_{i, p}(t)},
$$

where an additional weight $q_{i, p}$ is attached to each particle. This implies that each particle has a lower mass. The total mass is $\sum_{i=1}^{I_{p}(t)} q_{i, p} w_{i, p}(t)$. During the numerical experiments, we will use weights $q_{p}$ independent of both the specific $i$-th particle of population $p$ and of the time.

Further, we initialize the environment as follows: two straight vessels at $x=20 \Delta x$ and $x=40 \Delta x$, corresponding to a moderate vascular density of $50 \mathrm{~cm}^{2} / \mathrm{cm}^{3}$ (see [44]). The latter results in average oxygen concentrations, in the sense that cells are proceeding through the cell cycle at a speed, which is approximately half maximal. More details concerning realistic vascular densities and oxygen concentrations can be found in the supplementary material provided with [44].

The macroscopic equations are simulated using a simple Euler discretization for the time derivative and a second order central finite volume to discretize the spatial derivative. In the first three experiments, we have chosen for an explicit method. Further, the linear systems originating from the reaction-diffusion PDEs modeling the environment are solved using a conjugate gradient algorithm ( [28]). The default choice of discretization, time-step and number of cells can be found in table 3 .

The discussions corresponding to each of the individual experiments are organized as follows. First we consider the evolution of the population densities and the environment. Afterwards, we take a closer look at the variance with and without variance reduction. The results of the experiments are obtained by averaging out over 100 realizations.

Remark 1 (Color code). During the numerical experiments, we adopt the following color code to describe the different quantities. A colormap from white (low) towards gray (high) is used for the mean population densities (both normal and cancer) and the variance on the corresponding densities.

REMARK 2 (units). We use minutes as default time unit and $\mathrm{cm}$ as the spatial unit. Those will be omitted in the figure titles for compactness.

\begin{tabular}{|c|ccc|c|}
\hline Parameter & Normal & Cancer & EC & units \\
\hline$D_{p}$ & 0 & $5 \times 10^{-9}$ & $1 \times 10^{-8}$ & $\mathrm{~cm}^{2} / \mathrm{min}$ \\
$I_{p}(0)$ & 2000 & 200 & 0 & $\#$ particles \\
$q_{i, p}$ & 1 & 0.5 & 1 & dimensionless \\
$\delta t$ & 30 & 30 & 30 & min \\
$n_{p, \max }$ & 1 & 2 & 2 & $\#$ particles \\
$\Delta x$ & $4 \times 10^{-3}$ & $4 \times 10^{-3}$ & $4 \times 10^{-3}$ & $\mathrm{~cm}$ \\
$a$ & $0.5 \Delta x$ & $0.25 \Delta x$ & $\mathrm{~cm}$ \\
$b$ & $0.5 \Delta x$ & $0.05 \Delta x$ & $\mathrm{~cm}$ \\
\hline \multicolumn{5}{|c}{ TABLE 3} \\
Default parameter set used for the numerical experiments
\end{tabular}

4.1. A small-scale experiment with a static vasculature. In figure 2 , we have plotted the population density of the normal and cancer tissue, along with the 
oxygen concentration at time $t=5.76 \times 10^{4} \mathrm{~min}$. The tumor immediately influences
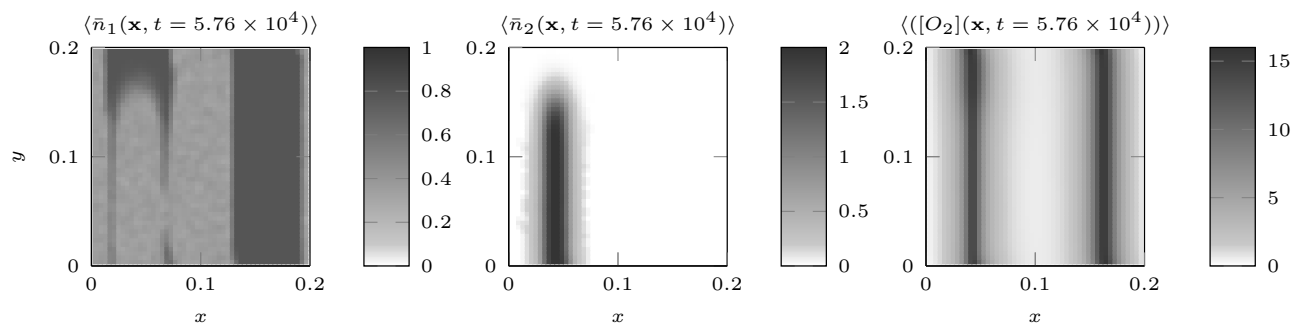

FIG. 2. Mean cellular densities -normal tissue: (left panel), cancer cell density: (middle panel) - and mean oxygen concentration (right panel) calculated using variance reduction at time $t=5.76 \times 10^{4} \mathrm{~min}$.

the normal tissue in the sense that a significant amount of normal cells die in this cancerous region. They literally have to make place for the growing tumor. Apart from apoptosis due to competition from cancer cells, cells also die due to lack off oxygen in between the two existing vessels. In the meantime, the tumor grows along the vessel until the tissue is locally saturated, meaning that $\sum_{p \in\{p=1, \ldots P\}} n_{p}(\mathbf{x}, t)>n_{p, \max }$. The high birth rate can be explained by the high oxygen concentration. Furthermore, the cells also diffuse in the other directions due to the random Brownian motion. This evolution can also be seen as an illustration of the "go or grow"-paradigm, which is identified as an important characteristic of the aggressiveness of the tumor [31, 25]. This process continues until the tissue is fully saturated along the leftmost vessel. Note that none of the cells is able to cross the low oxygen zone. They would all die due to the hypoxic environment.

We investigate how the population densities shown in figure 2 influence the variance. Figure 3 illustrates the elements contributing to the variance (see equation (28)). We first consider the variance with and without reduction in more detail. Combining the definitions of both variance and $n_{p}, \bar{n}_{p}$ yields:

$$
\begin{aligned}
\operatorname{Var}\left[n_{p}(\mathbf{x}, t)\right] & =\operatorname{Var}\left[n_{p}^{c}(\mathbf{x}, t)+R_{p}(\mathbf{x}, t)\right], \\
& =\operatorname{Var}\left[n_{p}^{c}(\mathbf{x}, t)+\operatorname{Var}\left[R_{p}(\mathbf{x}, t)\right]+2 \operatorname{Cov}\left(n_{p}^{c}(\mathbf{x}, t), R_{p}(\mathbf{x}, t)\right),\right. \\
\operatorname{Var}\left[\bar{n}_{p}(\mathbf{x}, t)\right] & =\operatorname{Var}\left[\tilde{n}_{p}^{c}(\mathbf{x}, t)\right]+\operatorname{Var}\left[R_{p}(\mathbf{x}, t)\right]+2 \operatorname{Cov}\left(\tilde{n}_{p}^{c}(\mathbf{x}, t), R_{p}(\mathbf{x}, t)\right),
\end{aligned}
$$

the variance on the reaction field (left), the variance on the corresponding control densities - without variance reduction $n_{2}^{c}$ and with variance reduction $\tilde{n}_{2}^{c}-($ middle) and the covariance between the reactions and the control densities (right). We compare the results without (first row) and with variance reduction (second row).

The variance on the reaction field is stretched along the leftmost vessel, as is the tumor itself. To explain the pattern in more detail, we have to compare the variance on the reaction field with the corresponding density. The variance is especially high just next to the largest concentration of the tumor, where the concentration of reactions is high due to the combined effect of the relatively high number of cancer cells and the fact the tissue is not fully saturated yet. Note that the variance on the reaction field does not depend on the variance reduction, since it is fully determined by the results of the agent-based simulation.

However, the image is completely different for the variance on the corresponding control densities. Observing the middle picture on the first row leads to the conclusion that the noise is dominated by random motion since the variance is larger in the 
middle of the tumor than at the border where most of the reactions take place. In contrast, after applying variance reduction the variance is fully determined by the reactions, implying that the variance is mostly filtered by the algorithm.

The above analysis (see figure 3 ) of the evolution of the variance clearly demonstrates the strong correlation between the oxygen concentration and the variance on the population densities. A more detailed view of the evolution can be found in the supplementary material (see section 6.2 for further details). To illustrate the performance of
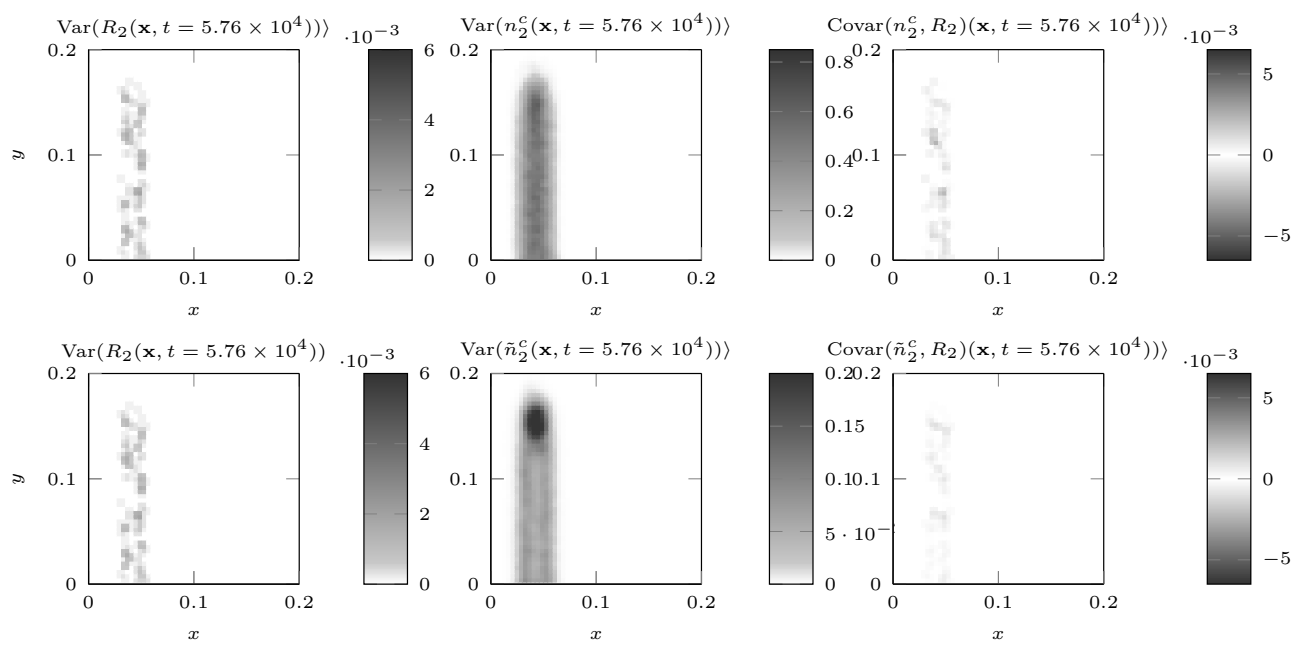

FIG. 3. Evolution of the factors contributing $-\operatorname{Var}\left(R_{2}(\mathbf{x}, t)\right)($ left $), \operatorname{Var}\left(n_{2}^{c}(\mathbf{x}, t)\right)$ (middle) and $\operatorname{Covar}\left(\left(R_{2}, n_{2}^{c}\right)(\mathbf{x}, t)\right)$ (right) - to variance on mean cancer cell density with (second row per time) and without variance reduction at time $t=5.76 \times 10^{4} \mathrm{~min}$.

the algorithm in another way, we have taken some slices - at $y=0.04 \mathrm{~cm}, y=0.1 \mathrm{~cm}$ and $y=0.14 \mathrm{~cm}$ respectively - of the cancer cell density at $t=5.76 \times 10^{4} \mathrm{~min}$. In figure 4 they are plotted along with their $95 \%$ confidence interval. The results based on the full stochastic model are plotted in black, while the results using the variance reduction algorithm are colored in gray. It can easily be seen that there is indeed a significant reduction and that the results using the variance reduction algorithm are consistent with the original results in the sense that they closely approximate the solution from the full stochastic model and that the variance is reduced significantly.

4.2. Experiment on a larger domain. As pointed out before, our latticefree approach allows to rescale the system in a straightforward way. Since the cost mainly depends on the number of particles and only marginal on the the domain size, it is possible to consider to perform a similar experiment on a rescaled (coarser) grid. To illustrate this we perform the simulation with $\Delta x=\Delta y=1.26 \times 10^{-2} \mathrm{~cm}$, corresponding to a domain of $0.4 \mathrm{~cm}^{2}$, corresponding with an upscaling of a factor 10. The normal tissue initially consists of $2 \times 10^{4}$ particles and a tumor of 1000 cells. According to the rescaled domain the maximal number of cells per volume was also scaled with a factor 10. In figure 5 we have plotted the evolution of the cellular distributions of the different cell types and the corresponding oxygen concentration. From the plot in the left column, one can see that normal cells are multiplying along the rightmost vessel since the left vessel is fully occupied by the tumor and there is not enough space for both the tumor and normal cells. In the rest of the domain the 

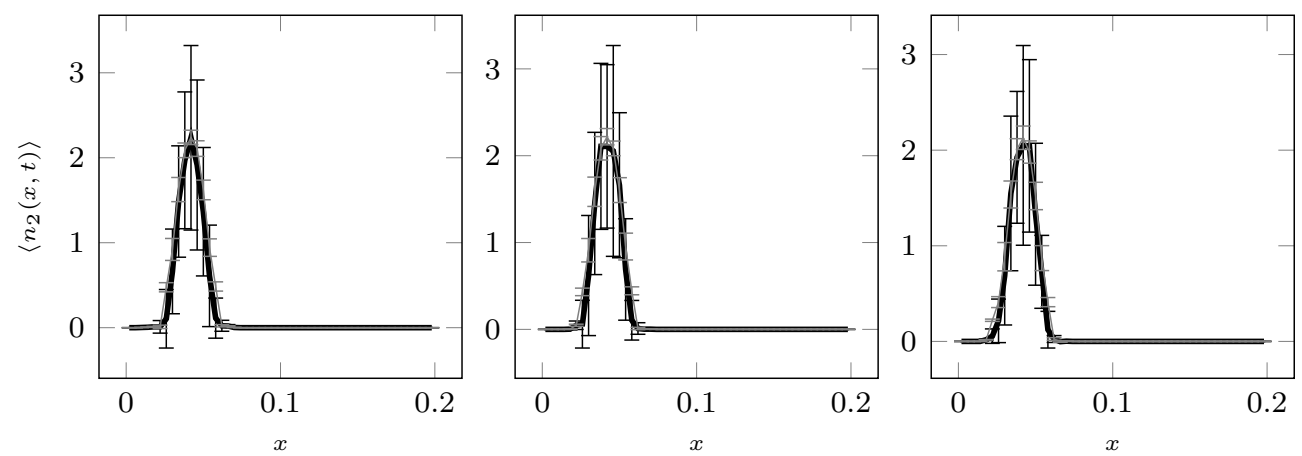

FIG. 4. Mean cancer cell density and corresponding $95 \%$ confidence interval at time $t=$ $5.76 \times 10^{4} \mathrm{~min}$ at $y=0.04 \mathrm{~cm}$ (left), $y=0.1 \mathrm{~cm}$ (middle) and $y=0.14 \mathrm{~cm}$ (gray) with (black) and without variance reduction (red).

normal tissue is reduced to a minimal level due to lack of oxygen and the influence of the tumor.
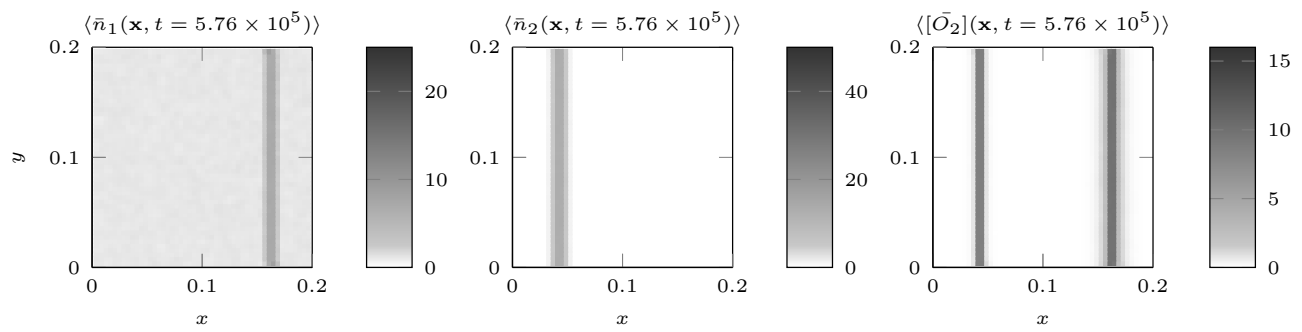

FIG. 5. Mean population densities and oxygen distribution in a large scale setting at time $t=5.76 \times 10^{5} \mathrm{~min}$.

In figure 6, we examine the influence of the variance reduction algorithm on the variance on the resulting tumor cell density as a function of time. Comparing the variance plot with (right panel) and without (left panel) give rise to the observation that the algorithm again yields a reduction of the variance both in the center and at the border of the tumor. This implies that the border of the tumor can be estimated in a more accurate, which determines the harshness of the tumor.
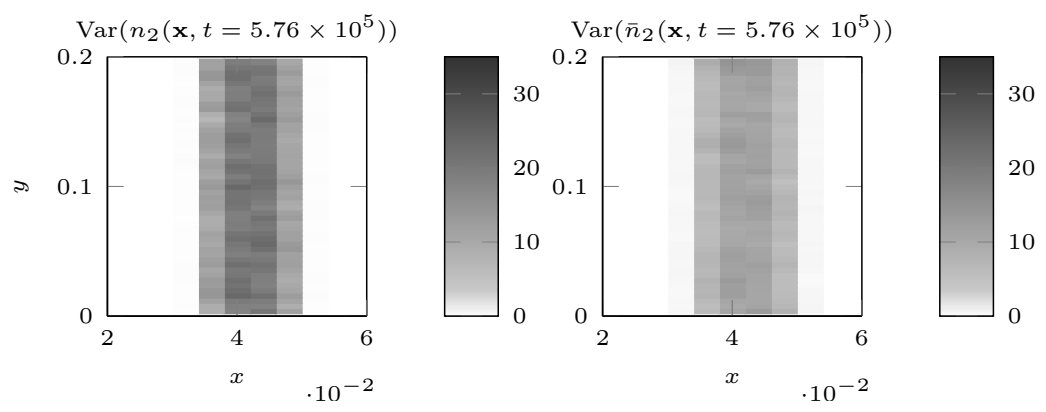

FIG. 6. Evolution of the variance on the mean cancer cell density in a large-scale setting with and without variance reduction at time $t=5.76 \times 10^{5}$ min, zoomed in on the left vessel. 
4.3. Variance reduction for sprouting angiogenesis. As a last experiment we will examine the performance of the algorithm in the case where the vasculature is also updated dynamically according to the model outlined in the section 2. A small tumor mass of initially 100 cells - sampled from a uniform distribution, with parameters $a_{2}=0.3 \Delta x, b_{2}=0.1 \Delta x$. The population density is discretized with 200 cells, i.e. $q_{i, 2}=0.5$. Further, we have chosen $D_{2}=1 \times 10^{-8} \mathrm{~cm}^{2} / \mathrm{min}$. The other parameters are set to the default values outlined in table 3 . In contrast to the previous experiments, the cancer cells are now able to cause extension of the vascular network according to their needs. The resulting oxygen distribution reveals a strong correlation with the cancer cell distribution itself, meaning that the tumor is fully vascularized now and can grow further. A small fraction of the tumor even managed to reach the second vessel supported by some new branches in the vascular network created in response to the high VEGF gradients. Next, we investigate how the
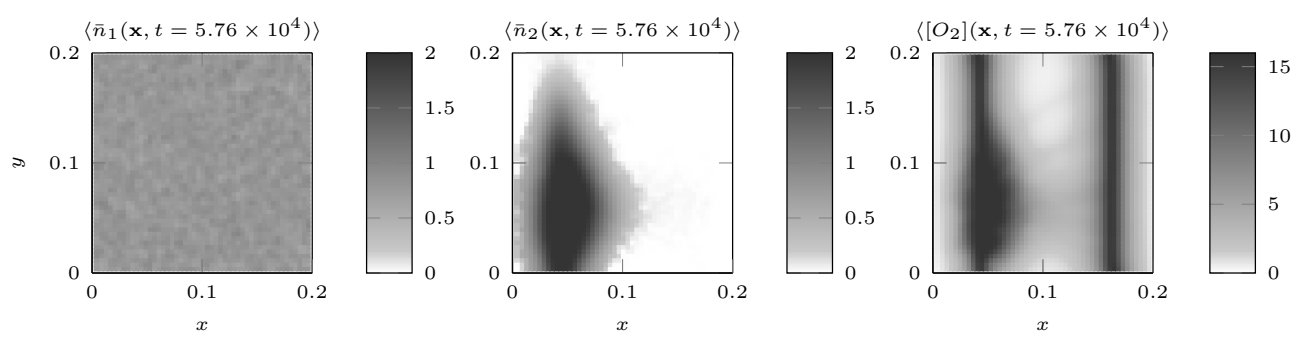

FIG. 7. Mean cellular densities and oxygen distribution at time $t=5.76 \times 10^{4}$ min with dynamical vasculature

variance reduction algorithm is performing in this setting of dynamic vascularization. In figure 8 , we have plotted the variance on the mean cancer cell density with $\left(\overline{n_{2}}(\mathbf{x}, t)\right)$ variance reduction on the right and and without variance reduction on the left.
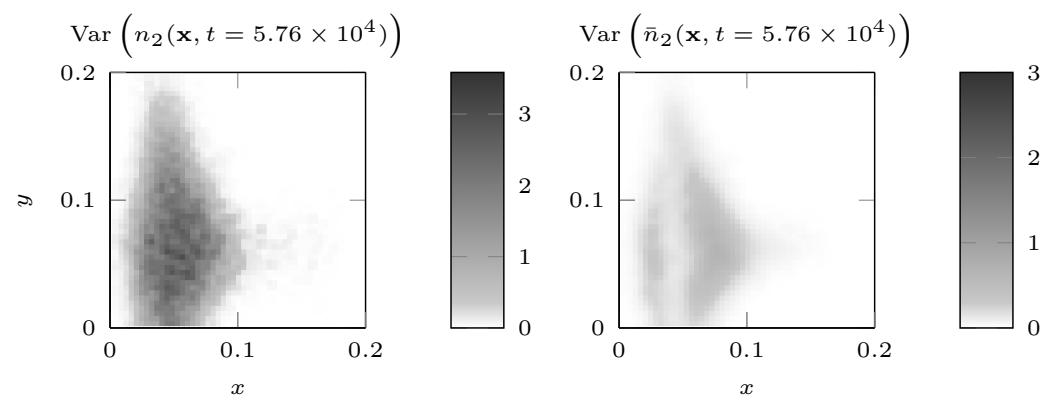

FIG. 8. Variance with and without variance reduction on the mean cancer cell density with a dynamic vasculature at time $t=5.76 \times 10^{4} \mathrm{~min}$

A comparison of the two variance plots in figure 8 leads to the conclusion that the variance is reduced everywhere. Along the leftmost vessel, the algorithm was even able to eliminate the noise completely. Indeed, the tissue is saturated here, so no new cells are born here. Besides, there is enough oxygen available to avoid apoptosis. Just outside this zone of maximal saturation the tissue is not so dense giving rise to more births and a higher level of noise here. In this region, the noise is proportional to the local density itself. 
4.4. Fast diffusing cancer cells. Motivated by the hypothesis that the diffusion coefficient can be related to the aggressiveness of the tumor, we investigate the situation where cancer cells have a higher diffusion coefficient. Swanson et al. have shown [60] that glioma's with a higher diffusion coefficient have a higher probability to cause metastases, which is obviously an important characteristic for the longterm survival probability of the patient. Apart from the modified diffusion coefficient $D_{2}=5 \times 10^{-7} \mathrm{~cm}^{2} / \mathrm{min}$, we adopt the same initial configuration as in the previous experiment. Note that the simulations are performed with a smaller time-step $(\delta t=0.3 \mathrm{~min})$ in order to fulfill the CFL-condition corresponding to the macroscopic equation.

In figure 9 , the evolution of both the normal tissue and the tumor are shown along with the local oxygen concentration at $t=1.152 \times 10^{3} \mathrm{~min}$. As in the previous experiment, the normal tissue density is the result of the cell deaths due to the presence of the tumor, while the normal cells are more sensitive to hypoxic environment. The tumor, on the other hand, has diffused through the normal tissue significantly on this short timescale, without consuming too much oxygen. Indeed, the cancer cells have already covered a large distance within a rather short time interval, meaning that the tumor exhibits the go-phenotype, rather than the grow-phenotype as it was the case in the first experiment. As can be observed from the leftmost picture in figure 9, only a relatively small fraction of the normal cells died. However, this scenario is potentially dangerous since it can stay more or less invisible for a long time and as soon as the tumor reaches a vessel it is possible that cells invade a vessel and give rise to metastatic spread of the cancer.

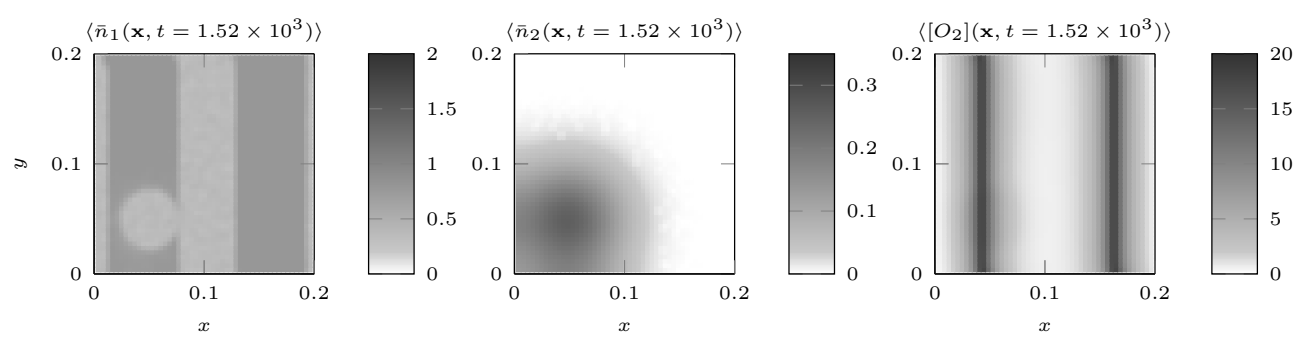

FIG. 9. Evolution of the cellular populations as a function of time (at time $t=1.52 \times 10^{3} \mathrm{~min}$, with fast diffusing cancer cells.

As before, we also examine the variance on the mean cancer cell density with and without variance reduction. Without variance reduction, the resulting variance is proportional to the density itself, suggesting that the variance is mainly caused by the random jumps. Obviously, the latter will be higher in zones with more cells. When variance reduction is applied, the variance is reduced with at least a factor 100 point-wise and moreover the plot reveals a clear pattern, which is again related to the oxygen concentration.

5. Discussion. We developed a novel variance reduction technique specifically suited to reduce the noise of agent-based models with birth and death events, as it is the case in our model for tumor growth. We proved that the algorithm outlined in section 3 gives rise to an unbiased estimator and the variance is determined by the births and deaths. The performance was illustrated numerically in different possible regimes characterizing different aspects of tumor growth such as sprouting angiogenesis, highly diffusive cancer cells and large-scale systems. The proposed algorithm 

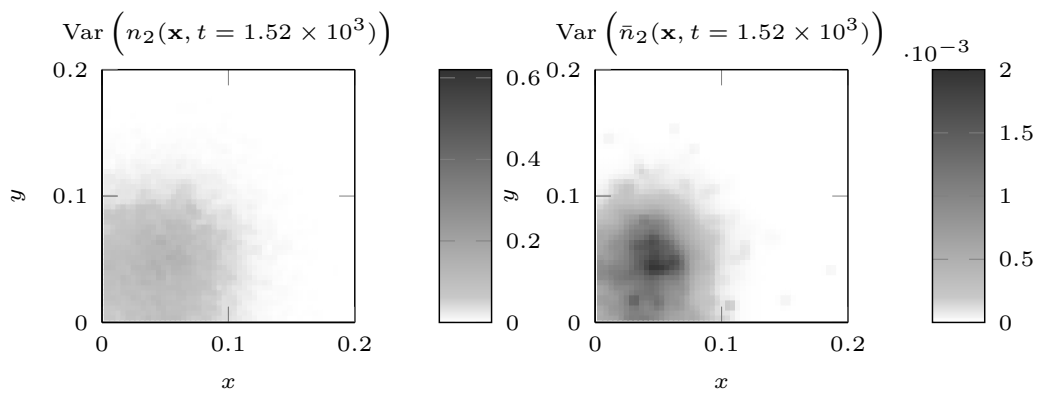

FIG. 10. Evolution of the variance on the mean cancer cell density with and without variance reduction at time $t=1.152 \times 10^{3} \mathrm{~min}$.

is based on the idea of control variates, since the evolution of the system without reactions is known deterministically via the macroscopic equation (13).

A valuable extension would be to combine this algorithm with other variance reduction techniques such as importance sampling. It is self-evident that an accurate and efficient simulation of all the different aspects of the system is crucially important for the reliability of the system as a whole. Apart from that, we will also extend our model with important features such as haptotaxis in response to the extra-cellular matrix and include a more sophisticated model for stemcellness $[15,41,16]$ since it was identified as one of the hallmarks of cancer [30]. Another track worthwhile further investigation is to apply our technique to patient-specific data. For instance, patientspecific data, like MRI-images or blood parameters, could be used as a specific initial configuration [39]. Finally, this algorithm can also be applied on related systems such as bone fracture healing and other application where we are interested in macroscopic behavior, but with agent-based features characterizing the dynamics.

\section{Supplementary material.}

6.1. Video 1. Evolution of the population densities in the small scale setting. Evolution of the mean normal and cancer cell density from time $t=0$ till time $t=5.76 \times 10^{4} \mathrm{~min}$. The initial configuration corresponds with the first numerical experiment. For the oxygen concentration we adopted a colormap with a range from white (low concentrations) towards black (high concentrations).

6.2. Video 2. Evolution of the variance in the small scale setting. Evolution of the variance on the mean normal and cancer cell density from time $t=0$ till time $t=5.76 \times 10^{4} \mathrm{~min}$. We again adopted a colormap with a range from white (low variance) towards black (high variance levels). 


\section{REFERENCES}

[1] B. D. AgudA, Modeling the cell division cycle, in Tutorials in Mathematical Biosciences III, Springer, 2006, pp. 1-22.

[2] J. A. Aguirre-Ghiso, Models, mechanisms and clinical evidence for cancer dormancy, Nature Reviews Cancer, 7 (2007), pp. 834-846.

[3] L. Albergante, J. Timmis, L. Beattie, and P. M. Kaye, A petri net model of granulomatous inflammation: implications for il-10 mediated control of leishmania donovani infection, PLoS Comput Biol, 9 (2013), p. e1003334.

[4] D. Alemani, F. Pappalardo, M. Pennisi, S. Motta, and V. Brusic, Combining cellular automata and lattice boltzmann method to model multiscale avascular tumor growth coupled with nutrient diffusion and immune competition, Journal of Immunological Methods, 376 (2012), pp. 55-68.

[5] A. R. A. Anderson, A. M. Weaver, P. T. Cummings, et Al., Tumor morphology and phenotypic evolution driven by selective pressure from the microenvironment., Cell, 127 (2006), pp. 905-15, doi:10.1016/j.cell.2006.09.042, http://www.ncbi.nlm.nih.gov/pubmed/ 17129778 .

[6] M. Bentele, I. Lavrik, M. Ulrich, S. Stösser, D. Heermann, H. Kalthoff, P. KramMER, AND R. EILS, Mathematical modeling reveals threshold mechanism in cd95-induced apoptosis, The Journal of cell biology, 166 (2004), pp. 839-851.

[7] K. Bentley, H. Gerhardt, And P. A. Bates, Agent-based simulation of notch-mediated tip cell selection in angiogenic sprout initialisation, J. Theor. Biol., 250 (2008), pp. 25-36, doi:10.1016/j.jtbi.2007.09.015.

[8] A. H. Berger, A. G. Knudson, And P. P. Pandolfi, A continuum model for tumour suppression., Nature, 476 (2011), pp. 163-9, doi:10.1038/nature10275, http:// www.pubmedcentral.nih.gov/articlerender.fcgi?artid=3206311\&tool=pmcentrez\& rendertype $=$ abstract.

[9] R. E. Caflisch, Monte carlo and quasi-monte carlo methods, Acta Numer., 7 (1998), pp. 1-49.

[10] Y. CAI, S. XU, J. WU, AND Q. LONG, Coupled modelling of tumour angiogenesis, tumour growth and blood perfusion, Journal of Theoretical Biology, 279 (2011), pp. 90-101.

[11] P. Carmeliet, Angiogenesis in life, disease and medicine, Nature, 438 (2005), pp. 932-936, http://dx.doi.org/10.1038/nature04478.

[12] P. Carmeliet And R. K. Jain, Angiogenesis in cancer and other diseases, nature, 407 (2000), pp. 249-257.

[13] A. Chakrabarti, S. Verbridge, A. D. Stroock, C. Fischbach, and J. D. Varner, Multiscale models of breast cancer progression, Annals of biomedical engineering, 40 (2012), pp. 2488-2500.

[14] M. A. Chaplain, S. R. McDougall, and A. Anderson, Mathematical modeling of tumorinduced angiogenesis, Annu. Rev. Biomed. Eng., 8 (2006), pp. 233-257.

[15] A. Cicalese, G. Bonizzi, C. E. Pasi, Et Al., The Tumor Suppressor p53 Regulates Polarity of Self-Renewing Divisions in Mammary Stem Cells, Cell, 138 (2009), pp. 1083-1095, doi:10.1016/j.cell.2009.06.048, http://dx.doi.org/10.1016/j.cell.2009.06.048.

[16] I. N. Colaluca, D. Tosoni, P. Nuciforo, ET AL., NUMB controls p53 tumour suppressor activity., Nature, 451 (2008), pp. 76-80, doi:10.1038/nature06412.

[17] G. D'Antonio, P. Macklin, and L. Preziosi, An agent-based model for elasto-plastic mechanical interactions between cells, basement membrane and extracellular matrix, Math. Biosci. Eng., 10 (2012), pp. 75-101, doi:10.3934/mbe.2013.10.75, http://www.aimsciences. org/journals/displayArticlesnew.jsp?paperID $=7991$.

[18] M. A. M. DE AGuiAR, E. RAuch, AND Y. BAR-YAm, Mean-field approximation to a spatial host-pathogen model, Physical Review E, 67 (2003), p. 047102.

[19] T. S. Deisboeck, Z. Wang, P. Macklin, et Al., Multiscale cancer modeling., Annu. Rev. Biomed. Eng., 13 (2011), pp. 127-55, doi:10.1146/annurev-bioeng-071910-124729, http:// www.ncbi.nlm.nih.gov/pubmed/21529163.

[20] G. Dimarco and L. Pareschi, Hybrid multiscale methods II. kinetic equations, Multiscale Model. Simul., 6 (2008), pp. 1169-1197.

[21] R. ERban and H. G. Othmer, From Individual to Collective Behavior in Bacterial Chemotaxis, SIAM J. Appl. Math., 65 (2004), pp. 361-391, doi:10.1137/S0036139903433232, http://epubs.siam.org/doi/abs/10.1137/S0036139903433232.

[22] G. P. Figueredo, T. V. Joshi, J. M. Osborne, H. M. Byrne, and M. R. Owen, On-lattice agent-based simulation of populations of cells within the open-source chaste framework, Interface focus, 3 (2013), p. 20120081.

[23] A. G. Fletcher, C. J. W. Breward, and S. Jonathan Chapman, Mathematical modeling 


\section{VARIANCE-REDUCED SIMULATION OF MULTISCALE TUMOR GROWTH MODELING21}

of monoclonal conversion in the colonic crypt, J. Theor. Biol., 300 (2012), pp. 118-133, doi:10.1016/j.jtbi.2012.01.021, http://dx.doi.org/10.1016/j.jtbi.2012.01.021.

[24] J. Galle, M. Loeffler, And D. Drasdo, Modeling the effect of deregulated proliferation and apoptosis on the growth dynamics of epithelial cell populations in vitro, Biophysical journal, 88 (2005), pp. 62-75.

[25] T. Garay, E. Juhász, E. MolnáR, ET AL., Cell migration or cytokinesis and proliferation?revisiting the "go or grow" hypothesis in cancer cells in vitro., Exp. Cell Res., 319 (2013), pp. 3094-103, doi:10.1016/j.yexcr.2013.08.018, http://www.sciencedirect.com/ science/article/pii/S0014482713003558.

[26] R. A. Gatenby And R. J. Gillies, Why do cancers have high aerobic glycolysis?, Nature Reviews Cancer, 4 (2004), pp. 891-899.

[27] H. Gerhardt, VEGF and endothelial guidance in angiogenic sprouting., Organogenesis, 4 (2008), pp. 241-246, doi:10.4161/org.4.4.7414.

[28] G. Guennebaud, B. JaCOB, And Others, Eigen v3. http://eigen.tuxfamily.org, 2010.

[29] D. Hanahan and R. A. Weinberg, The Hallmarks of Cancer, Cell, 100 (2000), pp. 57-70, doi:10.1016/S0092-8674(00)81683-9, http://www.sciencedirect.com/science/ article/pii/S0092867400816839.

[30] D. Hanahan and R. A. Weinberg, Hallmarks of cancer: the next generation., Cell, 144 (2011), pp. 646-74, doi:10.1016/j.cell.2011.02.013, http://www.sciencedirect.com/science/ article/pii/S0092867411001279.

[31] H. Hatzikirou, D. Basanta, M. Simon, et AL., 'Go or grow': The key to the emergence of invasion in tumour progression?, Math. Med. Biol., 29 (2012), pp. 49-65, doi:10.1093/imammb/dqq011.

[32] M. Hellström, L.-K. Phng, J. J. Hofmann, et Al., Dll4 signalling through Notch1 regulates formation of tip cells during angiogenesis., Nature, 445 (2007), pp. 776-780, doi:10.1038/nature05571.

[33] T. Hillen And K. J. Painter, A user's guide to PDE models for chemotaxis., J. Math. Biol., 58 (2009), pp. 183-217, doi:10.1007/s00285-008-0201-3, http://www.ncbi.nlm.nih. gov/pubmed/18626644.

[34] M. E. Hubbard And H. M. Byrne, Multiphase modelling of vascular tumour growth in two spatial dimensions., J. Theor. Biol., 316 (2013), pp. 70-89, doi:10.1016/j.jtbi.2012.09.031, http://www.sciencedirect.com/science/article/pii/S0022519312005097.

[35] L. Jakobsson, C. A. Franco, K. Bentley, Et AL., Endothelial cells dynamically compete for the tip cell position during angiogenic sprouting., Nat. Cell Biol., 12 (2010), pp. 943-53, doi:10.1038/ncb2103, http://www.ncbi.nlm.nih.gov/pubmed/20871601.

[36] Y. Kam, K. A. Rejniak, and A. R. Anderson, Cellular modeling of cancer invasion: integration of in silico and in vitro approaches, Journal of cellular physiology, 227 (2012), pp. 431-438.

[37] M. Kavousanakis, P. Liu, A. Boudouvis, ET Al., Efficient coarse simulation of a growing avascular tumor, Phys. Rev. E, 85 (2012), pp. 1-11, doi:10.1103/PhysRevE.85.031912, http://link.aps.org/doi/10.1103/PhysRevE.85.031912.

[38] C. Lemieux, Monte Carlo and Quasi-Monte Carlo Sampling, Springer New York, 2009, doi:10.1007/978-0-387-78165-5, http://link.springer.com/10.1007/978-0-387-78165-5.

[39] P. Macklin, M. E. Edgerton, A. M. Thompson, ET Al., Patient-calibrated agentbased modelling of ductal carcinoma in situ (DCIS): from microscopic measurements to macroscopic predictions of clinical progression., J. Theor. Biol., 301 (2012), pp. 122-40, doi:10.1016/j.jtbi.2012.02.002, http://www.sciencedirect.com/science/article/ pii/S0022519312000665.

[40] N. V. Mantzaris, S. Webb, and H. G. Othmer, Mathematical modeling of tumor-induced angiogenesis, Journal of mathematical biology, 49 (2004), pp. 111-187.

[41] S. J. Morrison And J. Kimble, Asymmetric and symmetric stem-cell divisions in development and cancer., Nature, 441 (2006), pp. 1068-1074, doi:10.1038/nature04956.

[42] M. M. Olsen and H. T. Siegelmann, Multiscale Agent-based Model of Tumor Angiogenesis, Procedia Comput. Sci., 18 (2013), pp. 1016-1025, doi:10.1016/j.procs.2013.05.267, http:// www.sciencedirect.com/science/article/pii/S1877050913004109.

[43] M. R. Owen, T. Alarcón, P. K. Maini, et AL., Angiogenesis and vascular remodelling in normal and cancerous tissues., J. Math. Biol., 58 (2009), pp. 689-721, doi:10.1007/s00285-008-0213-z, http://www.ncbi.nlm.nih.gov/pubmed/18941752.

[44] M. R. Owen, I. J. Stamper, M. Muthana, ET AL., Mathematical modeling predicts synergistic antitumor effects of combining a macrophage-based, hypoxia-targeted gene therapy with chemotherapy., Cancer Res., 71 (2011), pp. 2826-37, doi:10.1158/0008-5472.CAN-10-2834, http://www.pubmedcentral.nih.gov/articlerender.fcgi?artid=3527892\&tool=pmcentrez\& 
rendertype $=$ abstract.

[45] H. Perfahl, H. M. Byrne, T. Chen, V. Estrella, T. Alarcón, A. Lapin, R. A. Gatenby, R. J. Gillies, M. C. Lloyd, P. K. Maini, ET Al., Multiscale modelling of vascular tumour growth in 3d: the roles of domain size and boundary conditions, PloS one, 6 (2011), p. e14790.

[46] J. Poleszczuk And H. Enderling, A high-performance cellular automaton model of tumor growth with dynamically growing domains, Applied mathematics, 5 (2014), p. 144.

[47] G. A. Radtke and N. G. Hadjiconstantinou, Variance-reduced particle simulation of the Boltzmann transport equation in the relaxation-time approximation, Phys. Rev. E, 79 (2009), p. 56711, doi:10.1103/PhysRevE.79.056711, http://link.aps.org/doi/10.1103/ PhysRevE.79.056711.

[48] K. A. Rejniak and A. R. Anderson, Hybrid models of tumor growth, Wiley Interdisciplinary Reviews: Systems Biology and Medicine, 3 (2011), pp. 115-125.

[49] H. RIEGER AND M. WelteR, Integrative models of vascular remodeling during tumor growth, Wiley Interdisciplinary Reviews: Systems Biology and Medicine, 7 (2015), pp. 113-129.

[50] T. Roose, S. Chapman, And P. Maini, Mathematical Models of Avascular Tumor Growth, SIAM Rev., 49 (2007), pp. 179-208, doi:10.1137/S0036144504446291, http://epubs.siam. org/doi/abs/10.1137/S0036144504446291.

[51] M. Rousset And G. SAmaey, Simulating individual-based models of bacterial chemotaxis with asymptotic variance reduction, Math. Model. Methods Appl. Sci., 23 (2013), pp. 21552191, doi:10.1142/S0218202513500292, http://www.worldscientific.com/doi/abs/10.1142/ S0218202513500292.

[52] K. Schleich And I. N. Lavrik, Mathematical modeling of apoptosis, Cell Communication and Signaling, 11 (2013), pp. 1-7, doi:10.1186/1478-811X-11-44, http://dx.doi.org/10.1186/ 1478-811X-11-44.

[53] M. Scianna And L. Preziosi, Multiscale developments of the cellular potts model, Multiscale Modeling \& Simulation, 10 (2012), pp. 342-382.

[54] J. G. Scott, A. G. Fletcher, C. L. Timlin, D. Basanta, A. R. Anderson, and P. K. MAINI, Towards patient-specific biology-driven heterogeneous radiation planning: using a computational model of tumor growth to identify novel radiation sensitivity signatures., in 9th European Conference on Mathematical and Theoretical Biology, 2014.

[55] J. A. Sherratt And M. A. Chaplain, A new mathematical model for avascular tumour growth, Journal of mathematical biology, 43 (2001), pp. 291-312.

[56] S. L. Spencer And P. K. Sorger, Measuring and modeling apoptosis in single cells, Cell, 144 (2011), pp. 926-939.

[57] F. Spill, P. Guerrero, T. Alarcon, et Al., Mesoscopic and continuum modelling of angiogenesis, J. Math. Biol., (2014), pp. 1-48, doi:10.1007/s00285-014-0771-1, arXiv:arXiv:1401.5701v1.

[58] A. M. Stein, T. Demuth, D. Mobley, et Al., A mathematical model of glioblastoma tumor spheroid invasion in a three-dimensional in vitro experiment, Biophys. J., 92 (2007), pp. 356-365.

[59] S. H. Strogatz, Exploring complex networks, Nature, 410 (2001), pp. 268-276.

[60] K. R. Swanson, Quantifying glioma cell growth and invasion in vitro, Math. Comput. Model., 47 (2008), pp. 638-648, doi:10.1016/j.mcm.2007.02.024, http://www.sciencedirect.com/ science/article/pii/S0895717707001744.

[61] A. Szabó And R. M. Merks, Cellular potts modeling of tumor growth, tumor invasion, and tumor evolution, Frontiers in oncology, 3 (2013), p. 87.

[62] J. J. Tyson And B. Novak, Regulation of the eukaryotic cell cycle: molecular antagonism, hysteresis, and irreversible transitions., J. Theor. Biol., 210 (2001), pp. 249-63, doi:10.1006/jtbi.2001.2293, http://www.ncbi.nlm.nih.gov/pubmed/11371178.

[63] M. G. Vander Heiden, L. C. Cantley, and C. B. Thompson, Understanding the warburg effect: the metabolic requirements of cell proliferation, science, 324 (2009), pp. 1029-1033.

[64] J. Walpole, J. A. Papin, And S. M. Peirce, Multiscale computational models of complex biological systems, Annual review of biomedical engineering, 15 (2013), p. 137.

[65] K. P. Wilkie and P. Hahnfeldt, Mathematical models of immune-induced cancer dormancy and the emergence of immune evasion, Interface Focus, 3 (2013), p. 20130010.

[66] S. M. Wise, J. S. Lowengrub, H. B. Frieboes, et Al., Three-dimensional multispecies nonlinear tumor growth-I Model and numerical method., J. Theor. Biol., 253 (2008), pp. 524-43, doi:10.1016/j.jtbi.2008.03.027, http://www.ncbi.nlm.nih.gov/pubmed/18485374. 